\title{
Effects of a Physical Activity and Brain Exercise Program on Cognitive Ability of Healthy Thai Elders
}

\section{Mayuree LEETHONG-IN", Sirimart PIYAWATTANAPONG, Sawitree SOMMONGKOL, Supavadee THIENGTHAM and Nutda KUMNIYOM}

Gerontological Nursing Department, Faculty of Nursing, Khon Kaen University, Khon Kaen 40002, Thailand

('Corresponding author's e-mail: mayureekku@gmail.com)

Received: 21 April 2017, Revised: 12 December 2017, Accepted: 4 January 2018

\begin{abstract}
This study used a quasi-experimental single-group time series design to compare the effects of a physical activity and brain exercise program on the cognitive ability of healthy Thai elders. It was conducted from December 2015 to May 2016. A purposive sample of 23 people of over 60 years of age was used. The research instruments consisted of 1) the Mini-Mental State Examination Thai 2002 (MMSE-Thai 2002), 2) the Montreal Cognitive Assessment (MoCA, Thai version), and 3) the physical activity and brain exercise program devised for this research. The program components were validated and reviewed by 3 experts and yielded a content validity index (CVI) of 0.96. Participants attended the program 8 times $(3 \mathrm{~h}$ each) over 5 consecutive weeks. Subsequently, participant follow-up progress reviews were done in weeks 10,14, and 18. Data were analyzed using descriptive statistics and ANOVA with Wilcoxon matched-pairs signed-rank test and repeated measures ANOVA. Results revealed that the average score for cognitive ability (Mental State and cognitive function) in weeks 10 and 14 and at follow-up in week 18 were statistically significantly higher $(p<.05)$ than the baseline. Recommendation: the physical activity and brain exercise program could improve cognitive ability and should be included in health promoting activities in elders' clubs or senior schools in the future.
\end{abstract}

Keywords: Physical activity, brain exercise program, cognitive ability, healthy elders

\section{Introduction}

Dementia is an increasingly common condition in the elderly. The World Health Organization [1] reports that there are 47.5 million people with dementia worldwide, and there is an annual increase of 7.7 million new cases. This number is estimated to double by the year 2030. Likewise, the Thai National Health Examination Survey of 2008 - 2009 found 12.8 percent of the elderly, or about 880,000 persons, were affected by dementia, with the incidence doubling within successive 5 year age groups. The $60-69$ age group had a 7.1 percent incidence, and this increased to 32.5 percent in those above 80 years of age [2]. It is estimated that dementia prevalence in Thai elders will rise to 1.1 million in 2030 and to 2.0 million by 2050 [3]. The impacts of dementia do not affect only the patients, but also affect caregivers/families, both economically and socially, depending on symptom severity [4,5].

In early stage dementia, there is a change in the connections between the hippocampus and the prefrontal cortex, leading to changes in cognitive abilities, including losses in short-term memory, attention, language, orientation, calculation, spatial ability, and executive function [6], with further declines as aging continues [7]. The volume of caudate and putamen drops by about 3 percent every ten years, resulting in a decrease in working memory. The gap between the lobes of the brain becomes wider 
http://wjst.wu.ac.th

[8]. Hippocampus volume also declines, so attention and explicit executive function are also affected $[9,10]$. A study with healthy Thai elders revealed low scores in 5 cognitive functions; attention and calculation, recall, repetition, writing, and visuo-constructional skills [11]. If dementia in the elderly can be delayed by one year, its prevalence will drop by nearly one million; if it is delayed for 2 years, the number of new cases will fall by more than 2 million [12]. The prevention of dementia in the early stage will help delay brain degeneration [13]; thus, maintaining cognitive function in elders is important.

Cognitive stimulation in healthy elders can be provided by a variety of fun activities that focus on cognitive stimulation and social interaction. These activities can be performed by the elderly themselves or by interacting with others in small groups [14,15], with the intent of improving or maintaining cognitive ability [16] or to prevent or delay its worsening [17-19]. In addition, activities such as Tai Chi $[20,21]$ and physical training [22] also affect cognitive ability, especially memory [16,20,23-25].

It has been shown that combined cognitive and physical activity for a period of 5 to 30 weeks and 5 years can increase cognitive function in older persons, with cognitive activities preceding physical activity exercises $[20,22,26]$. Furthermore, elders attending physical activity programs, combined with cognitive training programs, had higher post-program cognitive scores at 1-year follow-ups compared to those who received only cognitive training programs [26].

Researchers have investigated and conducted effective programs to enhance cognitive function, with healthy Thai elders using a number of significant activities [25,27-29]. These activities included mostly cognitive stimulation activities, such as those with a focus on memory [27-29], attention [28,29], and executive function [25,27]. A previous Thai study combined physical activity with a cognitive training program with mildly cognitive impaired participants over 50 years of age [30]. Studies focusing on rehearsal activities, such as in exercise books, are still rare [25]; many studies showed that memory scores increased. However, orientation, attention, recall [27], and executive function did not increase [29]. It is considered that the existing level of knowledge of interventions suggests the need for more research to explore the impact of such a program on the cognitive functions of Thai elders.

According to the Atkinson-Shiffrin model [31], memory has 3 components; encoding, storage, and retrieval. The sequences of information flow through sensory memory, working memory with rehearsal and, finally, encoding and storage in the long-term memory for retrieval. Neurobic exercise refers to the integrated activities of the 5 physical senses and of emotional sense in daily chores, which activate underused nerve pathways and create direct connections in the cerebral cortex that can improve memory [32]. Social networking activities and positive emotions during social interactions may benefit cognitive functioning [33]. Furthermore, cognitive activities that consist of multiple activities, flexibility, and tasks of daily living likely enhance cognitive function more than a single activity. Therefore, integrating knowledge based on the Atkinson-Shiffrin model, Tai Chi, neurobic exercise, and social networking, especially interactions with others via mobile phone application (such as the INE application), may improve healthy Thai elder cognitive ability. The aim of this study was to investigate whether a combined physical activity and brain exercise program could promote cognitive ability in healthy Thai elders.

\section{Materials and methods}

\section{Design and setting}

The study used a single-group time series design to investigate the effect of an integrated physical activity and brain exercise program on a group of 23 healthy participants aged over 60 . The study was conducted between December 2015 and May 2016 within the municipal district of Khon Kaen Province, Northeastern Thailand.

\section{Participants and recruitment}

There were 23 participants, all over 60 years of age. Most participants were female $(82.6 \%) ; 52.2$ $\%$ were in the 60 - 69 years age group, and $43.5 \%$ had at least primary education. Participants' demographics are presented in Table 1. 
http://wjst.wu.ac.th

Table 1 Participants' demographics $(n=23)$.

\begin{tabular}{llc}
\hline Variables & & $\mathbf{n}(\mathbf{\%})$ \\
\hline Age & $60-69$ yr & $12(52.2)$ \\
& $\geq 70$ yr & $11(47.8)$ \\
\hline Gender & Men & $4(17.4)$ \\
& Women & $19(82.6)$ \\
\hline Marital status & Single & $3(13.0)$ \\
& Married & $7(30.4)$ \\
& Widow & $13(56.6)$ \\
\hline Educational status & Primary Education & $10(43.5)$ \\
& Secondary Education & $4(17.4)$ \\
& Higher Education & $9(39.1)$ \\
\hline
\end{tabular}

Participants were recruited via social media and advertising boards. Sixty potential participants initially volunteered and were enrolled, prior to a screening evaluation. After they were informed about the study, and had signed a consent form, demographic and health status data were collected, including data on age, gender, occupation, career, address, membership, chronic disease history, exercise behavior, and prior brain exercise program attendance. Participants completed screening assessments, which took approximately $1-1.5 \mathrm{~h}$. Fifty of the initial sixty volunteers passed the screening using the following inclusion criteria: being over 60 years of age; having a depression score of less than 7 points; having an activity of daily living score of at least 12 points; having a Time up and Go Test of less than $30 \mathrm{~s}$; having a Mini-Mental State Examination Thai 2002 (MMSE-Thai 2002) score of at least 17 points for those with primary school education, and at least 22 for those with higher than primary school education [34]; being able to use the LINE application on a mobile phone, having no illness or disease that affects body movement or cognitive ability, and having no history of drugs that interfere with nervous system.

A sample size calculation was performed, informed by previous studies. We used an expected effect size of 0.55 of MMSE improvement after the cognitive stimulation program. Using a power estimate of $80 \%$, and a confidence interval of $95 \%$, the estimated sample size was 22 participants. We added $10 \%$ (2 participants) to allow for possible loss or drop outs [35], giving a preferred sample size of 24.

Twenty-four of the fifty potential participants who passed the screening process were then purposively selected to participate. Of these, 1 was eventually excluded, due to incomplete study participation, giving an effective sample of 23

\section{Intervention: Physical activity and brain exercise program}

The physical activity and brain exercise program, modified from a previous study [30], had 5 components; 1) provision of knowledge, 2) physical activity including stretching, Tai Chi, dance, and a 9square table, 3) cognitive stimulation through orientation and reminiscence, 4) multisensory stimulation with post-session home practice rehearsal via 5 exercise books, and 5) ongoing group stimulation through the cell-phone LINE group application "FitKai Brain Exercise". Themed sessions involved memory, attention, calculation, language, visual-spatial functions, and executive functions. The program was validated by review with 3 experts with a content validity index level of 0.96. Program sessions were facilitated by nurses or nursing students who had attended a program training workshop. Facilitators supported, motivated, encouraged, provided resources, and checked the exercise books pre-post each session. The eight 3-hour sessions were conducted over 5 consecutive weeks. Follow-up progress reviews were done in weeks 10,14 and 18 .

\section{Data collection}

Two outcome measures were used in this study: the Mini-Mental State Examination Thai 2002 (MMSE-Thai 2002) and the Montreal Cognitive Assessment (MoCA, Thai version). Baseline measures 
were taken and follow-up progress reviewed at 10, 14 and 18 weeks. We used the MMSE-Thai 2002, a version translated and validated in 2002. It consists of orientation, registration, attention/calculation, recall, naming, repetition, verbal command, written command, writing, and visuo-construction items. The total score was 30 points. The Cronbach's alpha coefficient was .71. The Thai version of MoCA was employed to assess the cognitive function on visuospatial/executive, naming, memory, attention, language, abstraction, delayed recall, and orientation tasks [36]. The total score was 30 points (a score of 25 points or higher was considered normal).

Descriptive statistics were used to calculate participant demographics. The MMSE and MoCA scores were tested for normal distribution by the Shapiro-Wilk test. If the outcomes between the baseline and weeks 10, 14, and 18 were normally distributed, they were compared by Repeated Measures ANOVA, and Bonferroni technique was used for pairwise comparison. If the data were not normally distributed, Wilcoxon matched-pairs signed rank test was used, with a significance level set at .05. All analyses were performed by SPSS.

\section{Ethical review}

The research protocol was reviewed and approved by the Human Research Ethics Committee of Khon Kaen University (HE582279).

\section{Results and discussion}

The MMSE and MoCA levels at weeks 10, 14, and 18 were significantly higher than the baseline (Table 2), with a $p$-value of $<0.001$ and $<0.05$, respectively. At week 18, the median MMSE level increased from 26.00 at baseline to 29.00, while the MoCA also increased from 21.13 at baseline to 25.30. Clock drawing, one of the MoCA items, is used for measuring spatial dysfunction and monitoring cognitive change. This visuospatial/executive score at week 10, 14 and 18 was significantly higher than the baseline, with a $p$-value $<0.05$. Figure 1 is an example of improvement by one participant in terms of clock drawing.

Table 2 Median score of the Mini-Mental State Examination Thai 2002 (MMSE-Thai 2002) scores and mean score of Montreal Cognitive Assessment (MoCA) scores at week 10, week 14, and week 18 compared with baseline $(\mathrm{n}=23)$.

\begin{tabular}{lcc}
\hline & $\begin{array}{c}\text { MMSE } \\
\text { Median (IQR)* }\end{array}$ & $\begin{array}{c}\text { MoCA } \\
\text { Mean (SD)** }\end{array}$ \\
\hline Baseline & $26.00(3.50)$ & $21.13(4.00)$ \\
Week 10 & $28.00(2.00)^{\mathrm{a}}$ & $23.00(4.08)^{\mathrm{b}}$ \\
Week 14 & $28.50(2.00)^{\mathrm{a}}$ & $24.52(3.15)^{\mathrm{b}}$ \\
Week 18 & $29.00(1.00)^{\mathrm{a}}$ & $25.30(3.28)^{\mathrm{b}}$ \\
\hline
\end{tabular}

*Analysis by Wilcoxon matched-pairs signed rank test; ** Analysis by Repeated Measures ANOVA with Bonferroni; ${ }^{\mathrm{a}}$ indicated $p$-value less than $0.001 ;{ }^{\mathrm{b}}$ indicated $p$-value less than 0.05 . 


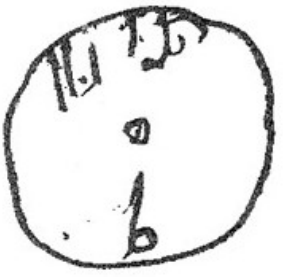

Baseline

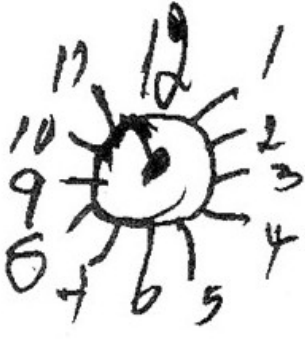

Week 10

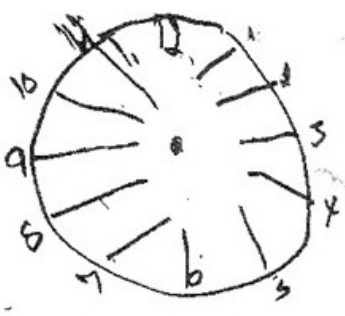

Week 14

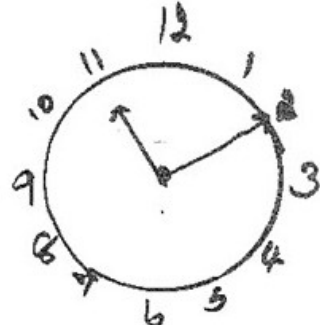

Week 18

Figure 1 Example of one participant's improvement in clock drawing at baseline, week 10, week 14, and week 18.

The physical activity and brain exercise program increased cognitive ability in the MMSE and the MoCA in healthy Thai elders when comparing from the baseline to an 18-week post-intervention follow-up. The median score of the MMSE scores and the mean score of the MoCA scores increased by 11.54 and $19.74 \%$, respectively. This is higher than the score reported in a Brazilian study conducted in healthy elderly (MMSE score $-0.26 \%$ ) [37], using a neurobic exercise study with 10 activities for 5 weeks (MMSE score $3.97 \%$ ) [28]. Furthermore, in a previous Thai study, the MoCA score improved after 3 months by $13.58 \%$ [30]. Our study indicated that the combined physical activity and brain exercise supported cognitive function more than by doing only physical activity or brain exercise, especially when the social activities were engaged with interesting activities [38]. There was a focus on social activities which were fun, and which encouraged the participants to do activities and use LINE group applications in their own homes. The elders also exchanged experiences, leading to selfesteem, motivation, encouragement, and positive relationships among them.

In the present study, the practice was introduced in a gradual manner, in 8 sessions of $3 \mathrm{~h}$ per week over 5 weeks, with "reboots" done in week 10 and week 14. It also led to learning and collaboration in practice. When the activities were successfully done, the experience and the confidence in the elders' ability were raised, for which they could continue to practice. Although there were no sessions during weeks 14 - 18, they were still reinforced through LINE group activity and appointments for "friendhelping-friend activities". A program activities review in weeks 10 and 14 showed the participants continuously performed the activities and were confident in their own abilities. In addition, they supported the success of friends and their successful experiences. Similarly, participants had time to practice, think, and continuously stimulate cognitive functions [16]. Our results were similar to a study of nursing home elders who received cognitive training 2 times per week for 7 weeks. Their average cognitive function score was higher than that of the control group [23].

Previous reports also found increased cognitive function over periods of 5 to 30 weeks and 5 years from cognitive activities without physical activities [20,22,26]. Our participants differed from previous studies in some respects; we enrolled healthy participants of 60 years or older, who lived in the municipality, and used the LINE applications on smart phone. Another Thai study had used participants of 50 years of age or older in a 3-month program [30]. Another study had participants with suspected dementia who received training 3 times a week for 5 consecutive weeks [29], and a Korean study included persons with mild amnestic cognitive impairment who participated twice per week over 12 weeks [39].

The low-cost program described in this study was found to be practically feasible in community settings, e.g., in local temples. The cultural appropriateness of using familiar daily activities, related workbooks for home work exercises, and LINE application support enhanced the elder persons' motivation to continue in the program. The project team's concern and respect for the elder persons also contributed to the participants' strong engagement in the program. 
http://wjst.wu.ac.th

\section{Limitations of the study}

The study was limited, since there was no comparison with a control group. Also, a threat to internal validity, namely "testing", may occur, since the educational level of most of the elderly tested was higher than primary school. Therefore, when they were assessed repeatedly, they may remember the questions, and this may affect the dependent variables.

\section{Conclusions and recommendations}

The program mainly consisted of cognitive stimulation, such as reality orientation, reminiscence therapy, neurobic exercise, physical activity, and homework, to stimulate cognitive functions. The program activities were designed for repetitive practice on specific cognitive tasks. The participants would gain knowledge on how to live a healthy lifestyle to prevent dementia. However, enhancing the roles of caregivers and family in the program in the future and testing it with rural communities is recommended. The program is shown to be beneficial, is not complicated, and has no detrimental consequences. Our findings suggest that multiple approaches and multi-cognitive domains may produce the most benefits in healthy elderly, rather than focusing on one approach or a single domain. Further study should investigate other outcomes, such as physical, mental, and social dimensions. Moreover, longer-term effects should be explored, as well as having a comparison group to better understand the unique impact of the program.

\section{Acknowledgements}

We would like to thank all of the participants and facilitators who participated in this study. Funding for this study was supported by grants from the Bureau of Academic Services and the Faculty of Nursing, Khon Kaen University, Thailand. We acknowledge, with a deep sense of gratitude, Associate Professor Dr. John F. Smith, for the time devoted to editing this article.

\section{Reference}

[1] World Health Organization. Dementia a Public Health Priority, Available at: http://www.who.int/mental_health/neurology/dementia/dementia_thematicbrief_executivesummary. pdf, accessed January 2016.

[2] P Ekachampaka and N Wattanamano. Health Status and Health Problem of Thai. In: S Wibulpolprasert (ed). Thai Health Profile 2008-2010 (in Thai). The War Veterans Organization of Thailand, Bangkok, 2011, p. 147-61.

[3] Alzheimers Disease International. Dementia in the Asia Pacific Region, Available at: https://www.alz.co.uk/adi/pdf/Dementia-Asia-Pacific-2014.pdf, accessed December 2016.

[4] N Srinim. Problem and coping experience of caregivers who provided care for dementia patients at home. J. Ment. Health. Thai. 2005; 23, 61-71.

[5] P Ondee, R Panitrat, K Pongthavornkamol, V Senanarong, TA Harvath and D Nittayasudhi. Factors predicting depression among caregivers of persons with dementia. Pacific Rim Int. J. Nurs. Res. 2013; 17, 167-80.

[6] DE Vance, YD Eaves, NL Keltner and TS Struzick. Practical implications of procedural and emotional religion activity therapy for nursing. J. Gerontol. Nurs. 2010; 36, 22-9.

[7] D Maillet and MN Rajah. Age-related changes in frequency of mind-wandering and task-related interferences during memory encoding and their impact on retrieval. Memory 2013; 21, 818-31.

[8] MP Mattson. Cellular and Neurochemical Aspects of the Aging Human Brain. In: JB Halter, JG Ouslander, ME Tinetti and S Studenski (eds.). Hazzard's Geriatric Medicine and Gerontology. $6^{\text {th }}$ eds. Mc Graw-Hill, New York, 2009, p. 739-50.

[9] KR Daffner. Promoting successful cognitive aging: A comprehensive review. J. Alzheimers Dis. $2010 ; 19,1101-22$.

[10] C Grady. The cognitive neuroscience of ageing. Nat. Rev. Neurosci. 2012; 13, 491-505. 
http://wjst.wu.ac.th

[11] UY Yaem and N Yaemsakul. A survey of dementia in the elderly in Damneonsadual district, Ratchaburi province (in Thai). Prim. Health. Care Div. J. 2013; 9, 29-31.

[12] W Muangpaisan. Risk Factors of Dementia and Prevention (part I), Available at: http://www.si.mahidol.ac.th/Th/department/psychiatrics/dept_article_detail.asp?a_id=415, accessed January 2016

[13] W Srithanyarat, P Arunsang, M Leethong-in, S Pitawattanapong and L Panpanich. Case Study Review and Synthesis of service System for Continuing Care of Older People: Case Study of Older People with Stroke and Dementia (in Thai). Khon Kaen, 2015.

[14] National Institute for Health and Care Excellence. Dementia: Supporting People with Dementia and Their Carers in Health and Social Care, Available at: https://www.nice.org.uk/guidance/cg42. accessed April 2016.

[15] A Kueider, K Bichay and G Rebok. Cognitive Training for Older Adults: What is It and Does It Work?, Available at: http://www.air.org/sites/default/files/downloads/report/CognitiveTraining forOlderAdults_Nov14.pdf, accessed December 2016.

[16] B Woods, E A Auirre, AE Spector and M Orrell. Cognitive stimulation to improve cognitive functioning in people with dementia. Cochrane Database Syst. Rev. 2012; 2, CD005562.

[17] L Clare and RT Woods. Cognitive training and cognitive rehabilitation for people with early-stage Alzheimer's disease: A review. Neuropsychol. Rehabil. 2004; 14, 385-401.

[18] S Tardif and M Simard. Cognitive stimulation programs in healthy elderly: A review. Int. J. Alzheimers. Dis. 2011; 2011, 378934.

[19] J Reijnders, C van Heugten and M van Boxtel. Cognitive interventions in healthy older adults and people with mild cognitive impairment: A systematic review. Ageing Res. Rev. 2013; 12, 263-75.

[20] JA Mortimer, D Ding, AR Borenstein, CD Carli, Q Guo, Y Wu, Q Zhao and S Chu. Changes in brain volume and cognition in a randomized trial of exercise and social interaction in a communitybased sample of non-demented Chinese elders. J. Alzheimers Dis. 2012; 30, 757-66.

[21] PM Wayne, JN Walsh, RE Taylor-Piliae, RE Wells, KV Papp, NJ Donovan and GY Yeh. Effect of Tai Chi on cognitive performance in older adults: Systematic review and meta-analysis. J. Am. Geriatr. Soc. 2014; 62, 25-39.

[22] WD Oswald, T Gunzelmann, R Rupprecht and B Hagen. Differential effects of single versus combined cognitive and physical training with older adults: The SimA study in a 5-year perspective. Eur. J. Ageing 2006; 3, 179.

[23] JLA Apostolo, DFB Cardoso, AI Rosa and C Paul. The effect of cognitive stimulation on nursing home elders: A randomized controlled trial. J. Nurs. Scholarsh 2014; 46, 157-66.

[24] JD Huntley, RL Gould, K Liu, M Smith and RJ Howard. Do cognitive interventions improve general cognition in dementia? A meta-analysis and meta-regression. BMJ Open 2015; 5, e005247.

[25] $\mathrm{T}$ Chattrawongwiwat, $\mathrm{W}$ Kong-in and $\mathrm{P}$ Thaniwattananon. Impacts of a memory-enhancing programme on perceived memory self-efficacy amongst elderly people (in Thai). Thai J. Nurs. Counc. 2013; 28, 98-108.

[26] J Rahe, A Petrelli, S Kaesberg, GR Fink, J Kessler and E Kalbe. Effects of cognitive training with additional physical activity compared to pure cognitive training in healthy older adults. Clin. Interv. Aging 2015; 10, 297-310.

[27] A Choombuathong, R Chaitiamwong, P Sanguansit and A Tothonglor. Effect of aerobic exercise on reaction time and memory in the elderly (in Thai). J. Heal. Sci. 2015; 24, 283-95.

[28] T Samantarat. The neurobics exercise for memory enhancement in elderly patients received services in diabetic and hypertension clinic of $\mathrm{Si}$ Piman community health center (in Thai). $J$. Baromarajonani Coll. Nurs. Bangkok 2015; 31, 106-20.

[29] P Chaiwong, P Rattakorn and P Mumkhetvit. Effects of cognitive training program on cognitive abilities and quality of life in elderly with suspected dementia (in Thai). Bull. Chiang Mai Assoc. Med. Sci. 2015; 48, 182-91.

[30] O Kuha and P Phongchaturawit. Cognitive Stimulation with Mild Cognitive Impairment in People (in Thai). Somdej Phranyanasawara the supreme Patriarch Elderly Center, Nonthaburi, 2016.

[31] RC Atkinson and RM Shiffrin. Human memory: A proposed system and its control processes. 
http://wjst.wu.ac.th

Psychol. Learn. Motivat. 1968; 2, 89-195.

[32] L Katz and M Rubin. Keep Your Brain Alive: 83 Neurobic Exercises to Help Prevent Memory Loss and Increase Mental Fitness. Workman Publishing Company, New York, 1999, p. 1-8.

[33] ST Charles and LL Carstensen. Social and emotional aging. Annu. Rev. Psychol. 2010; 61, 383-409.

[34] The Board Conducted a Preliminary Test of The Brain Condition Thai Test Performance. Primary Brain Thai MMSE-Thai 2002 (in Thai). Department of Medical Service, Nonthaburi, 2002.

[35] SK Grove. Sampling. In: SK Grove, N Burns and JR Gray (eds.).The Practice of Nursing Research, Appraisal, Synthesis, and Generation of Evidence. $8^{\text {th }}$ ed. Elsevier saunders, China, 2017, p. 329-63.

[36] S Hemrungrojn. Available at: http:/www.mocatest.org/wp-content/uploads/2015/tests-instructions/ MoCA-Instructions-Thai.pdf, accessed September 2015.

[37] TB Lima-Silva, AT Fabricio, LDSV Silva, GMD Oliveira, WTD Silva, PT Kissaki, APFD Silva, TF Sasahara, TN Ordonez, TBD Oliveira, FO Aramaki, A Buriti and MS Yassuda. Training of executive functions in healthy elderly: Results of a pilot study. Dement. Neuropsychol. 2012; 6, 3541.

[38] PD Bamidis, AB Vivas, C Styliadis, C Frantzidis, M Klados, W Schlee, A Siountas and SG Papageorgiou. A review of physical and cognitive interventions in aging. Neurosci. Biobehav. Rev. 2014; 44, 206-20.

[39] JH Jeong, HR Na, SH Choi, J Kim, DL Na, SW Seo, J Chin, SA Park, EJ Kim, HJ Han, SH Han, SJ Yoon, JH Lee, KW Park, SY Moon, MH Park, MS Choi, IW Han, JH Lee, JS Lee, YS Shim and JY Kim. Group- and home-based cognitive intervention for patients with mild cognitive impairment: A randomized controlled trial. Psychother. Psychosom. 2016; 85, 198-207. 